\title{
A Gaussian transition of an optical speckle field studied by the minimal spanning tree method
}

Olivier Vasseur

olivier.vasseur@onera.fr

Isabelle Bergoënd

Xavier Orlik
ONERA, Theoretical and Applied Optics Department, 91120 Palaiseau, France

ONERA, Theoretical and Applied Optics Department, 31055 Toulouse, France

We propose to study the Gaussian transition of an optical speckle field using the Minimal Spanning Tree method. We perform an analysis of the spatial intensity distribution and show that the maxima of intensity evolve from a cluster distribution in the strongly non Gaussian regime, to a gradient distribution around the transition and then approach the random distribution area when we tend to the Gaussian regime. In the generated minimal spanning trees, we observe that the standard deviation of the edges length exhibits a maximum around the Gaussian transition when about 4 correlation cells of the surface roughness are illuminated. [D0I: 10.2971/je0s.2010.10052]

Keywords: speckle, surface roughness, Gaussian transition, minimal spanning tree, statistical optics

\section{INTRODUCTION}

An optical speckle field [1] generated by the scattering from a rough surface can contain useful informations about the surface properties especially in the case the incident beam illuminates only a few correlation cells of the surface roughness $[2,3]$. However, working with a unique illuminating spot size in such a non Gaussian regime doesn't allow a full characterization of the surface roughness properties. Nevertheless, as it seems to be general in Physics, following a parameter along a transition brings much more information that an observation of the same parameter at a unique point of this transition. Thus, we have recently proposed the idea of studying the transition from the non Gaussian to the Gaussian regime of the speckle field in order to increase the amount of accessible information concerning the surface roughness [4]. Using a Gaussian correlated dephasing screen to modelize the surface roughness, we have shown that, by following such transition generated by a varying illuminating spot size, the speckle contrast was sufficient to discriminate between roughness values in the full range $[0 ; \lambda], \lambda$ being the wavelength of the monochromatic illuminating laser. On a practical point of view, such method based on the Gaussian transition study is expected to improve absolute surface roughness and correlation length measurements by speckle analysis [5]-[7].

The probability density function of intensity is helpful to characterize an optical speckle field, however it doesn't contain any information about the spatial distribution of the field. In this paper, we use a new sensitive criterion to characterize this spatial intensity distribution. Our approach is based on a specific graph constructed from the set of points of the local maxima of the intensity distributions in an observation plane. This graph, called Minimal Spanning Tree (MST) [8], allows us to deduce two parameters - the mean and the standard deviation of the edges length - that characterize the distributions of the points (ordered, random, cluster...). We present here the first results of speckle fields characterization obtained by the MST method and we apply it to the study of a Gaussian transition.

\section{GENERATION OF NON GAUSSIAN SPECKLE FIELDS}

The model consists first in the generation of a random rough surface modelized by a random correlated Gaussian phase screen that exhibits a Gaussian heights distribution. Then, in the case of a Gaussian beam illumination, we calculate the corresponding scattered field at a given distance using the Fresnel diffraction integral [9]. This model has been previously used for the study of the circular Gaussian transition of speckle fields in the near field region [4]. We just propose in this section a brief restatement of the main points.

The discretized and correlated profiles of the surfaces roughness are calculated by the following equation [10] :

$$
\left.\mathcal{S}(\xi, \eta)=F T^{-1}[\sqrt{F T[\mathcal{A}(\xi, \eta)}] \cdot F T[X(\xi, \eta)]\right]
$$

where :

$$
\mathcal{A}(\xi, \eta)=H_{R M S}^{2} \exp \left[-\frac{\xi^{2}+\eta^{2}}{L_{c}^{2}}\right],
$$

with :

- $H_{R M S}$ the standard deviation of the surface heights distribution that will be simply referred as "roughness" in the forthcoming sections 
- $L_{c}$ the correlation length of the surface heights distribution

- $X(\xi, \eta)$ an uncorrelated Gaussian random function with zero mean and standard deviation of unity.

FT denotes here a discrete bidimensional Fourier Transform.

The incident field characterized by a Gaussian amplitude distribution and a wavelength $\lambda=632 \mathrm{~nm}$ impacts the surface parallel to its normal. Its waist $\omega_{0}$ is chosen to be located on the mean level of the surface. The scalar field undergoes the dephasing associated to the random correlated Gaussian surface and the scattered field at the distance $z$ in the transverse plane $(x, y)$ is then calculated using the Fresnel diffraction integral :

$$
\tilde{E}_{S c}(x, y, z)=\iint_{\mathcal{S}} \tilde{E}(\xi, \eta) \cdot \tilde{\mathcal{G}}(x-\xi, y-\eta, z) d \xi d \eta,
$$

with $\tilde{\mathcal{G}}$ the propagator in the paraxial approximation :

$$
\tilde{\mathcal{G}}(x-\xi, y-\eta, z)=\frac{e^{i k z}}{i \lambda z} \exp \left[\frac{i k}{2 z}\left((x-\xi)^{2}+(y-\eta)^{2}\right)\right],
$$

and $\tilde{E}(\xi, \eta)$ the initial Gaussian beam of maximum amplitude unity that has been dephased according to the correlated surface profile $\mathcal{S}(\xi, \eta)$ :

$$
\tilde{E}(\xi, \eta)=\exp \left[-\frac{\xi^{2}+\eta^{2}}{\omega_{o}^{2}}\right] \cdot \exp [i k \mathcal{S}(\xi, \eta)] .
$$

The paraxial propagation of the scattered field is performed using the following Fourier Transforms :

$$
\tilde{E}_{s c}(x, y, z)=F T^{-1}[F T[\tilde{E}(\xi, \eta)] . F T[\tilde{\mathcal{G}}(x-\xi, y-\eta, z)]] .
$$

This calculation allows the determination of the complex scattered speckle field and its statistics even in the non Gaussian regime where the central limit theorem can't be applied [4].

\section{DESCRIPTION OF THE MINIMAL SPANNING TREE METHOD}

An edge-weighted linear graph $G=(X, E)$ is composed of a set of points $X=\left\{x_{1}, x_{2}, \ldots\right\}$ called nodes and a set of node pairs $E=\left\{\left(x_{i}, x_{j}\right)\right\}$ called edges, with a number called weight (the Euclidean distance in our case) assigned to each edge. A tree is a connected graph without closed loops. A Minimal Spanning Tree (MST) is a tree which contains all the nodes with a minimal sum of the edge weights [8]. It can be noticed that for a set of $N$ points (corresponding to a number of edges of $N-1$ ) which are randomly distributed with uniform intensity in a 2-dimensional region with an area of $A$, the total length of the MST is asymptotically given by $L_{G \infty}=\alpha_{2}(A N)^{1 / 2}$ where $\alpha_{2}$ depends on the problem solved [11]. So the mean length of an edge is given by:

$$
M_{A}=\alpha_{2} \frac{(A N)^{1 / 2}}{N-1}
$$

The area $A$ of the sampling window of a data set can be estimated by a normalization of the area $H$ of the convex hull of the data by the following relation:

$$
A=\frac{H}{1-(f / N)} \text {. }
$$

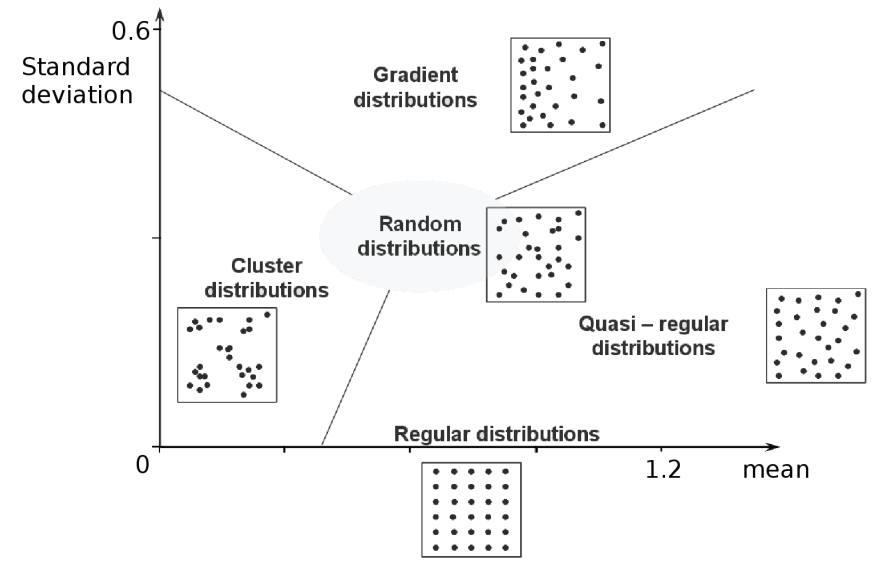

FIG. 1 Points distributions in the $(m, \sigma)$ plane. Random distributions are built by the use of probability density functions of the continuous uniform distribution to generate the coordinates of the points.

where $f$ is the number of faces of the convex hull [12].

In our study, the convex hull is the smallest square containing all the points. Depending on the starting point there may be more than one MST for a given set of points, but all of the MST's have the same length-edge histogram. The normalized values of the mean $m$ and the standard deviation $\sigma$ of the edges length can be used to characterize the distribution of points (ordered, random, cluster...) [13]. In the field of topographical analysis, this method presents the advantages of an high discrimination power and stability to characterize spatial point patterns [14]. In the $(m, \sigma)$ plane, all distribution of points can be plotted and easily compared with wellcharacterized distributions (for example, perfectly ordered with $\sigma=0$ or random ones with $m \approx 0.65$ and $\sigma \approx 0.30$ ) as shown in Figure 1.

\section{STUDY OF THE GAUSSIAN TRANSITION BY THE MINIMAL SPANNING TREE METHODS}

For a given set of roughness value $H_{R M S}$ and numbers $n_{c}$ of correlation cells illuminated by a Gaussian beam, we generate, as described in Section 2, 10 random surfaces and the corresponding 10 intensity distributions of the field in the observation plane at the distance $0,75 z_{R}$ from the surface, $z_{R}$ being the Rayleigh distance. From each intensity distribution, we detect the set of all the points representing a local maximum of the intensity distribution above a threshold. The MST is built on this set of points and provides the location of the spatial intensity maxima distribution in the $(m, \sigma)$ plane. Figure 2 presents the process along the Gaussian transition of a completely developed speckle $\left(H_{R M S}=\lambda\right)$, for intensity distributions obtained in the case of the following $n_{c}$ values : 0.5, 2 and 50 .

The windows of analysis has been defined in 2 different ways. When the same square sampling window is used to build the MST's of all the intensity images in the Gaussian transition, the influence of the extension of the speckle in the observation plane is taken into account. When the area of the window is estimated by the normalization of the convex hull of the 
(a)

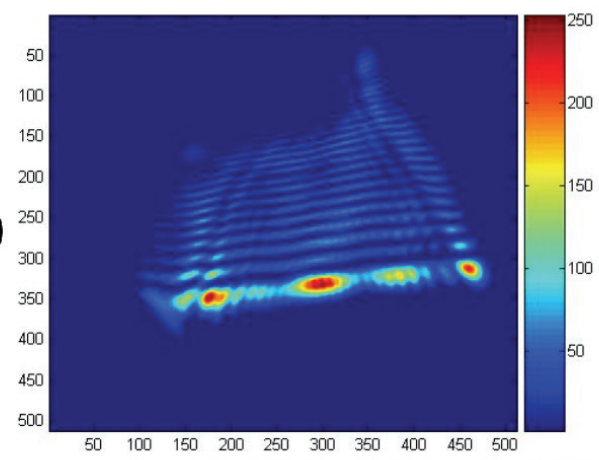

(b)

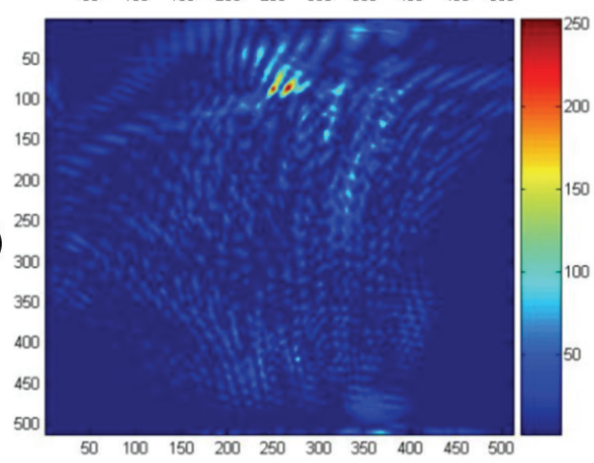

(c)

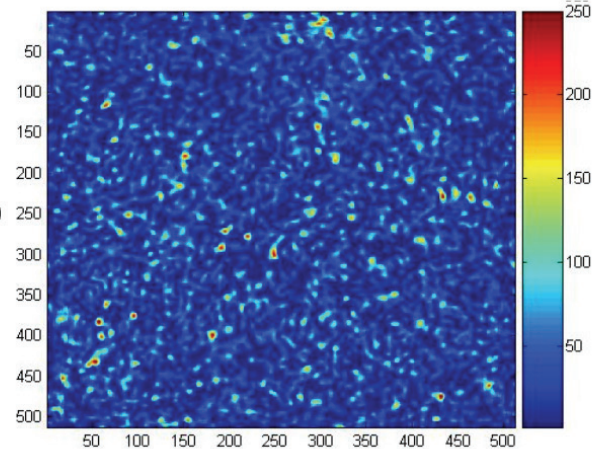

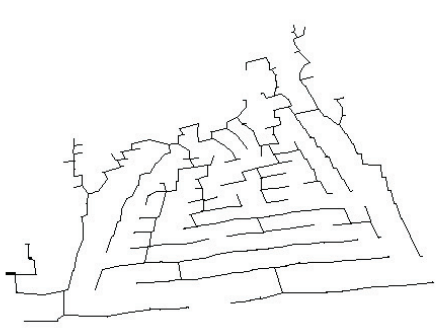
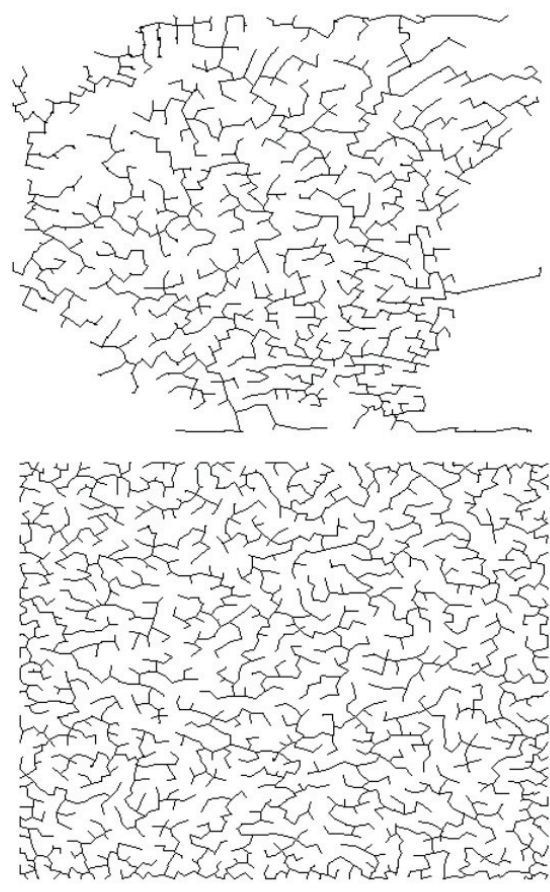

FIG. 2 Process analysis of the spatial intensity distribution by the MST. (a) (b) and (c) correspond respectively to $n_{c}=0.5,2$ and 50 illuminated correlation cells of the surface roughness. The 3 columns display from left to right : the intensity distribution in the observation plane, the points of local maxima and the corresponding MST.

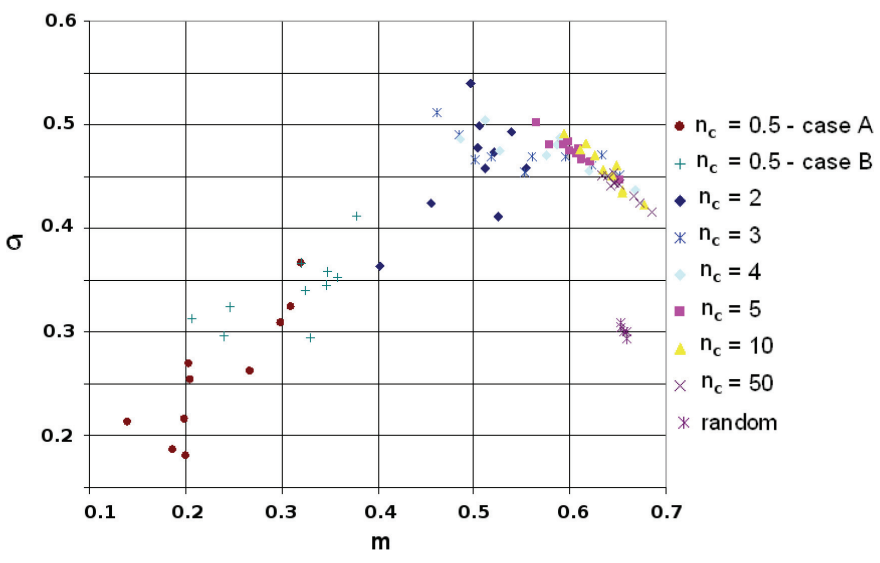

FIC. 3 Representation of the average $m$ and standard deviation $\sigma$ of MST's edges built on maxima intensity distributions in the Gaussian transition of a speckle field. $n_{c}$ corresponds to the number of illuminated correlation cells of the surface roughness. In case $A$, the same square windows of analysis is used whereas in case $B$, different normalized square windows are used for an intrinsic analysis. We observe a maximum of $\sigma$ around the Gaussian transition. data, we obtain an intrinsic analysis of the spatial distribution of points. The results obtained along the Gaussian transition in both cases are presented on Figure 3. All values of $n_{c}>0.5$ give the same result in the $(m, \sigma)$ plane regardless of the definition of the window of analysis. For the case $n_{c}=0.5$, when the sampling window is kept constant, the values of the mean length and of the standard deviation obtained (Figure 3, case A) are significantly lower than those calculated using the sampling window estimated by the normalization of the convex hull of the data (Figure 3, case B). This is due to the fact that the set of points of maximum intensity does not reach the border of the analysis window because of the highly non Gaussian illumination conditions giving rise to a more localized repartition of energy. Along the Gaussian transition, we can observe the evolution of the location in the $(m, \sigma)$ plane due to the increased number of illuminated correlation cells. In the strongly non Gaussian regime and according to the intrinsic quantitative analysis (Figure 3 for $n_{c}=0.5$, case B), we observe that the spatial distribution of the maxima of intensity corresponds to the cluster distribution area. When $n_{c}$ increases (until few units) and reaches the Gaussian transition, the values of $m$ and $\sigma$ increase to the gradient distribution area. For higher and increasing values of $n_{c}$, the location slowly tends 
to the random distribution area $(m \approx 0.65$ and $\sigma \approx 0.30)$. We point out that the $\sigma$ value exhibits a clear maximum $\left(n_{c} \approx 4\right)$ around the Gaussian transition.

\section{CONCLUSION}

We have proposed the first results of speckle fields characterization obtained by the use of the Minimal Spanning Tree method. The introduction of a criterion, namely the location in the $(m, \sigma)$ plane that corresponds to maximum intensity regions, have been used to perform a spatial intensity distribution analysis along the Gaussian transition of an optical speckle field. When we evolve from a strongly non Gaussian regime to the Gaussian one, this location evolves from a cluster distribution to a gradient distribution and then approaches a random distribution. Moreover, the MST method exhibits a maximum of the standard deviation of the edges length around the Gaussian transition when about 4 correlation cells of the surface roughness are illuminated. Both results provide a new and robust way to characterize the correlation length of a surface roughness and its illumination conditions. This method can find further applications such as the study of non circular speckle fields and the spatial distribution of phase singularities in the Gaussian transition. In a more general way, it can improve rough surface or multiple scattering media characterization by speckle field analysis.

\section{References}

[1] J. W. Goodman, Speckle phenomena in optics, theory and applications (Roberts and Company, Colorado, 2006).

[2] H. Fujii, "Contrast variation of non-Gaussian speckle" Opt. Act. 27, 409-418 (1980).

[3] M. Deka, S. P. Almeida, and H. Fujii, “Root-mean-square difference between the intensities of non-Gaussian speckle at two different wavelengths" J. Opt. Soc. Am. 71, 155-163 (1981).

[4] I. Bergoënd, X. Orlik, and E. Lacot, "Study of a circular Gaussian transition in an optical speckle field" J. Europ. Opt. Soc. Rap. Public. 3, 08028 (2008).

[5] H. Kadano, T. Asakura, and N. Takai, "Roughness and correlation length determination of rough-surface objects using speckle contrast" Appl. Phys. B 44, 167-173 (1987).

[6] H. Kadano, T. Asakura, and N. Takai, "Roughness and correlation length measurements of rough-surface objects using the speckle contrast in the diffraction field" Optik 80, 115-120 (1988).

[7] C. Cheng, C. Liu, N. Zhang, T. Jia, R. Li, and Z. Xu, “Absolute measurement of roughness and lateral-correlation length of random surfaces by use of the simplified model of image-speckle contrast" Appl. Opt. 41, 4148 (2002).

[8] C. Zhan, "Graph-theoretical methods for detecting and describing gestalt clusters" IEEE T. Comput. C-20, 68-86 (1971).

[9] R. Grella, "Fresnel propagation and diffraction and paraxial wave equation" J. Opt. 13, 367-374 (1982).

[10] A. K. Fung, and M. F. Chen, "Numerical simulation of scattering from simple and composite random surfaces" J. Opt. Soc. Am. A 2, 2274-2284 (1985).

[11] J. Beardwood, J. Halton, and J. Hammersley, "The shortest path through many points" P. Camb. Philos. Soc. 55, 299-327 (1959).

[12] R. Hoffman, and A. Jain, "A test of randomness based on the minimal spanning tree" Pattern Recogn. Lett. 1, 175-180 (1983).

[13] C. Dussert, G. Rasigni, M. Rasigni, and J. Palmari, “Minimal spanning tree: A new approach for studying order and disorder" Phys. Rev. B 34, 3528-3531 (1986).

[14] F. Wallet, and C. Dussert, "Comparison of spatial point patterns and processes characterization methods" Europhys. Lett. 42, 493498 (1998). 